\title{
On the Super Codes of the First Order Reed-Muller Code Based on m-Sequence Pairs
}

\author{
Ying $\mathrm{Xu}$, and Yuejun Wei \\ Department of Wireless Research \\ Huawei Technologies Co., Ltd, Shanghai, 201206, China \\ Email: \{eaglexu,weiyuejun\}@huawei.com
}

\author{
Yuhang Yang, and Wen Chen \\ Department of Electronic Engineering \\ Shanghai Jiao Tong University, Shanghai, 200240, China \\ Email: \{yhyang,wenchen\}@sju.edu.cn
}

\begin{abstract}
The super codes of the first order Reed-Muller code are widely used in the practical wireless communication systems, such as WCDMA and LTE. However, the super codes are usually obtained by exhaustive computer search, which involves huge computational cost. Therefore a systematic algorithm to construct the super codes beyond exhaustive computer search has been expected for long time. In this paper, we propose a systematic algorithm for the super codes construction base on the m-sequence pairs, which only involves some item permutation within the $m$-sequence. In addition, the constructed super codes outperform the conventional codes in terms of decoding error rate. Meanwhile, the super codes share the same characteristics as the conventional ones, such as being of arbitrary length and rate, and being efficiently decodable with Fast Hadamard Transform (FHT).
\end{abstract} FHT

Keywords-Super codes, Reed-Muller code, m-Sequence Pairs,

\section{INTRODUCTION}

The super codes of the first order Reed-Muller are a conventional error-correcting code, which can provide excellent performance when the code length is short. Therefore the super codes are widely used in wireless communication systems for short message coding. For examples, in WCDMA [1], super codes are used to encode TFCI (Transport Format Combination Indicator); in LTE [2], the super codes are used to encode PUCCH (Physical Uplink Control Channel) and PUSCH (Physical Uplink Shared Channel).

However, it is very difficult to construct the super codes of the first order Reed-Muller code with competitive performance in terms of decoding error rate. It is well known that the performance of the super codes is decided by the distribution of code distance, especially the minimum code distance [3]. Conventionally, the super codes with good distribution of code distance are usually obtained by exhaustive computer search [4], which, however, is much cost in computation and may miss some useful codes.

Up to now, there has been some analysis about the utilization of the super codes of Reed-Muller code. For example, they can be utilized to reduce the PAPR of OFDM signals [5]-[7]. Some researchers also have studied the weight distribution of the super codes of Reed-Muller code, including the first order Reed-Muller code [8]-[9] and the second order Reed-Muller code [10]. However, it has been nearly a decade that there is not substantial advance on the construction of super codes of the first order Reed-Muller code.

In this paper, we propose a systematic algorithm to construct the super codes of the first order Reed-Muller code based on m-sequence pairs, which can well control the minimum code distance. At the same time, it can get rid of the exhaustive computer search, and only involves some simple item permutation within the $\mathrm{m}$-sequence. The constructed super codes can offer better performance compared to the conventional ones. Specifically, we first investigate the equivalence of $\mathrm{m}$-sequences and Walsh sequences. Then we construct all $\mathrm{m}$-sequences from a generating polynomial pair and select some linearly independent $\mathrm{m}$-sequences from them. After that, item permutation is performed over the selected $\mathrm{m}$ sequences to construct the super codes. Finally, simulation results are presented to verify our construction.

\section{EQUIVALENCE OF m-SEQUENCE AND WALSH SEQUENCE}

\section{A. Theoretical Analysis}

The m-sequence is a kind of pseudo random sequences, and the Walsh sequence is a kind of orthogonal sequences [11]. It seems that they are not related to each other. However, a Walsh sequence can be constructed by permuting the items within a m-sequence [12]-[13].

Given a generating polynomial $f(x)$, we can construct a group of m-sequences based on $f(x)$. The generated $\mathrm{m}$ sequences are actually the circular sequences of each other. If the order of $f(x)$ is $n$, it will generate $2^{n}-1$ different $\mathrm{m}$ sequences of length $2^{n}-1$. We mark these $\left(2^{n}-1\right) \mathrm{m}$ sequences as $m_{i}=\left(m_{i}(t)\right)_{1 \leq t \leq 2^{n}-1}$, for $1 \leq i \leq 2^{n}-1$.

Since the order of $f(x)$ is $n$, there are at most $n$ linear independent sequences among $\left(m_{i}\right)_{1 \leq i \leq 2^{n}-1}$. Obviously, there are many different sets of such $n$ linear independent sequences. Suppose that $\left(p_{j}\right)_{1 \leq j \leq n}$ is a set of $n$ linear independent sequences selected from $\left(m_{i}\right)_{1 \leq i \leq 2^{n}-1}$. Then it is actually a $n \times\left(2^{n}-1\right)$ generating matrix $\left(p_{j}(t)\right)_{1 \leq j \leq n, 1 \leq t \leq 2^{n}-1}$. We now build the permutation pattern as 


$$
C(t)=\sum_{j=1}^{n} p_{j}(t) \times 2^{j-1}, \text { for } 1 \leq t \leq 2^{n}-1 .
$$

Then column permutation is performed to the matrix $\left(m_{i}(t)\right)_{1 \leq i \leq 2^{n}-1,1 \leq t \leq 2^{n}-1}$ according to permutation pattern $C(t)$, that is

$$
m_{i}^{\prime}(C(t))=m_{i}(t), 1 \leq i \leq 2^{n}-1,1 \leq t \leq 2^{n}-1 .
$$

At last, add digit " 0 " at the beginning of each $m_{i}^{\prime}$, and then $m_{i}^{\prime}$ becomes a sequences of length $2^{n}$ for $1 \leq i \leq 2^{n}-1$. These new $2^{n}-1$ sequences are actually the Walsh sequences of order $n$.

\section{B. An Example}

Take the generating polynomial $f(x)=x^{3}+x^{1}+1$ as an example. Then $n=3$ and the m-sequences $m_{i}$ can be generated by $f(x)=x^{3}+x^{1}+1$ as

$$
\begin{aligned}
& m_{1}=(0011101) \\
& m_{2}=(0100111) \\
& m_{3}=(0111010) \\
& m_{4}=(1001110) \\
& m_{5}=(1010011) \\
& m_{6}=(1101001) \\
& m_{7}=(1110100)
\end{aligned}
$$

Select $p_{1}=m_{1}, p_{2}=m_{2}, p_{3}=m_{4}$ to form a set of linear independent sequences, and calculate permutation pattern $C(t)$ as

$$
C(t)=\{4,2,1,5,7,6,3\} .
$$

After column permutation according to $C(t)$ and adding digit " 0 " at the beginning of each sequence, we have

$$
\begin{aligned}
& m_{1}^{\prime}=01010101 \\
& m_{2}^{\prime}=00110011 \\
& m_{3}^{\prime}=01100110 \\
& m_{4}^{\prime}=00001111 \\
& m_{5}^{\prime}=01011010 \\
& m_{6}^{\prime}=00111100 \\
& m_{7}^{\prime}=01101001
\end{aligned}
$$

Obviously, $m_{i}^{\prime}$ is a Walsh sequence of order 3 , for $1 \leq j \leq n$.

More introductions on the equivalence of $\mathrm{m}$-sequences and Walsh sequences can be found in [14]-[16].

\section{SUPER CODES CONSTRUCTION}

In this section, we will introduce the construction algorithm for super codes of the first order Reed-Muller code based on $\mathrm{m}$-sequence pairs.

\section{A. M-Sequence Pairs}

Given two m-sequences $a$ and $b$ of order $n$. If the crosscorrelation function $R_{a b}(\tau)$ of $a$ and $b$ is valued within

$$
\left\{2^{\lfloor(n+2) / 2\rfloor}-1,-1,-2^{\lfloor(n+2) / 2\rfloor}-1\right\},
$$

Then $a$ and $b$ form an m-sequence pair.

The m-sequence pair has good cross-correlation characteristics, and is usually used to construct Gold sequences [17]-[18]. In this paper, we utilize the cross-correlation characteristics of the m-sequence pair to construct the super codes of the first order Reed-Muller code with good performance and low decoding complexity.

\section{B. Construction Algorithm}

Step 1: Take an m-sequence pair generating polynomial $f_{1}(x)$ and $f_{2}(x)$, and generate the corresponding $\mathrm{m}$-sequences respectively. Mark the corresponding $\mathrm{m}$ sequences as $m_{i}, w_{i}$, for $1 \leq i \leq 2^{n}-1$.

Step 2: Select $n$ linear independent sequences from $\left(m_{i}\right)_{1 \leq i \leq 2^{n}-1}$, and mark them as $\left(p_{j}\right)_{1 \leq j \leq n}$. Similarly, choose $n$ linear independent sequences $\left(q_{j}\right)_{1 \leq j \leq n}$ from $\left(w_{i}\right)_{1 \leq i \leq 2^{n}-1}$. Note that $p_{j}$ and $q_{j}$ are selected from the same indexed msequences of $\left(m_{i}\right)_{1 \leq i \leq 2^{n}-1}$ and $\left(w_{i}\right)_{1 \leq i \leq 2^{n}-1}$.

Step 3: Calculate the permutation pattern $C(t)$ by

$$
C(t)=\sum_{j=1}^{n} p_{j}(t) \times 2^{j-1} .
$$

Step 4: Permute $p_{j}(t)$ and $q_{j}(t)$ according to $C(t)$, i.e.,

$$
\begin{aligned}
& p_{j}^{\prime}(C(t))=p_{j}(t), 1 \leq j \leq n, 1 \leq t \leq 2^{n}-1 \\
& q_{j}^{\prime}(C(t))=q_{j}(t), 1 \leq j \leq n, 1 \leq t \leq 2^{n}-1
\end{aligned}
$$

Step 5: Add digit " 0 " at the beginning of each $p_{j}^{\prime}$ and $q_{j}^{\prime}$. Then make a generating matrix

$$
G=\left[\begin{array}{ccc}
1 & \cdots & 1 \\
p_{1}^{\prime}(1) & \cdots & p_{1}^{\prime}\left(2^{n}-1\right) \\
\vdots & \cdots & \vdots \\
p_{n}^{\prime}(1) & \cdots & p_{n}^{\prime}\left(2^{n}-1\right) \\
q_{1}^{\prime}(1) & \cdots & q_{1}^{\prime}\left(2^{n}-1\right) \\
\vdots & \cdots & \vdots \\
q_{n}^{\prime}(1) & \cdots & q_{n}^{\prime}\left(2^{n}-1\right)
\end{array}\right],
$$

which can generate $\left(2^{n}, 2 n+1\right)$ linear block codes.

According to the analysis in section II, we can find that $p_{j}^{\prime}(t)$ is actually a Walsh sequence. Therefore, the constructed 
super codes can be decoded by using the Fast Hadamard Transform (FHT) [19]-[20], which can greatly reduce the decoding complexity.

As we know, the performance of linear block codes is totally determined by the distribution of code distance, and mainly influenced by the minimum code distance. The constructed super codes enjoy large minimum distance, which is summarized in the following table.

Table 1. Minimum distance of the proposed $\left(2^{n}, 2 n+1\right)$ codes

\begin{tabular}{|c|c|c|c|}
\hline Information Length & $\mathbf{1}$ & $\mathbf{2} \sim \boldsymbol{n + 1}$ & $\boldsymbol{n}+\mathbf{2} \sim \mathbf{2 n + 1}$ \\
\hline Minimum distance & $2^{n}$ & $2^{n-1}$ & $2^{n-1}-2^{\lfloor(n+2) / 2\rfloor-1}$ \\
\hline
\end{tabular}

\section{An Example}

For easy understanding, we present an example to demonstrate the construction algorithm. The parameters in the example come from the criteria of LTE [2].

Step 1: Let $n=5$. Take $f_{1}(x)=x^{5}+x^{2}+1$ and $f_{2}(x)=x^{5}+x^{4}+x^{2}+x^{1}+1$ as the generating polynomials of $\mathrm{m}$-sequence pairs, and construct the corresponding $\mathrm{m}$ sequences $m_{i}$ and $w_{i}$. Due to limited space, we will not list the $\mathrm{m}$-sequences here.

Step 2: Select the $1^{\text {st }}, 2^{\text {nd }}, 4^{\text {th }}, 8^{\text {th }}$ and $16^{\text {th }}$ sequences from $m_{i}$ and $w_{i}$ to form $\left(p_{j}\right)_{1 \leq j \leq 5}$ and $\left(q_{j}\right)_{1 \leq j \leq 5}$. It is easy to prove that either of $\left(p_{j}\right)_{1 \leq j \leq 5}$ or $\left(q_{j}\right)_{1 \leq j \leq 5}$ is linearly independent set. List $\left(p_{j}\right)_{1 \leq j \leq 5}$ and $\left(q_{j}\right)_{1 \leq j \leq 5}$ as follows.

$$
\begin{aligned}
& p_{1}(\mathrm{t})=0000101011101100011111001101001 \\
& p_{2}(\mathrm{t})=0001010111011000111110011010010 \\
& p_{3}(\mathrm{t})=0010000101011101100011111001101 \\
& p_{4}(\mathrm{t})=0100001010111011000111110011010 \\
& p_{5}(\mathrm{t})=1000010101110110001111100110100 \\
& q_{1}(t)=0000110101001000101111101100111 \\
& q_{2}(\mathrm{t})=0001011111011001110000110101001 \\
& q_{3}(\mathrm{t})=0010001011111011001110000110101 \\
& q_{4}(\mathrm{t})=0100010111110110011100001101010 \\
& q_{5}(\mathrm{t})=1000011010100100010111110110011
\end{aligned}
$$

Step 3: Calculate the permutation pattern $C(t)$ according to $p_{j}(t)$. Then we derive

$$
\begin{aligned}
C(t)= & \{16,8,4,2,1,27,22,11,30,15, \\
& 28,14,7,24,12,6,3,26,13,29, \\
& 21,17,19,18,9,31,20,10,5,25,23\}
\end{aligned}
$$

Step 4: Permute $p_{j}$ and $q_{j}$ according to the permutation pattern of $C(t)$, and get $p_{j}^{\prime}$ and $q_{j}^{\prime}$ as follows.

$$
\begin{aligned}
& p_{1}^{\prime}(\mathrm{t})=1010101010101010101010101010101 \\
& p_{2}^{\prime}(\mathrm{t})=0110011001100110011001100110011 \\
& p_{3}^{\prime}(\mathrm{t})=0001111000011110000111100001111 \\
& p_{4}^{\prime}(\mathrm{t})=0000000111111110000000011111111 \\
& p_{5}^{\prime}(\mathrm{t})=0000000000000001111111111111111 \\
& q_{1}^{\prime}(t)=1000111001000010111101100011101 \\
& q_{2}^{\prime}(t)=0110110010111110110001100001010 \\
& q_{3}^{\prime}(t)=0001100010110010101100111110011 \\
& q_{4}^{\prime}(t)=0010001101101000111011111010010 \\
& q_{5}^{\prime}(t)=0010100011100101110010001111101
\end{aligned}
$$

Obviously $p_{j}^{\prime}(t)$ is a Walsh sequence,

Step 5: Add digit " 0 " at the beginning of each $p_{j}^{\prime}$ and $q_{j}^{\prime}$. Then the generating matrix is

$$
G=\left[\begin{array}{c}
1 \cdots 1 \\
p_{j}^{\prime} \\
q_{j}^{\prime}
\end{array}\right]=\left[\begin{array}{l}
11111111111111111111111111111111 \\
01010101010101010101010101010101 \\
00110011001100110011001100110011 \\
00001111000011110000111100001111 \\
00000000111111110000000011111111 \\
00000000000000001111111111111111 \\
01000111001000010111101100011101 \\
00110110010111110110001100001010 \\
00001100010110010101100111110011 \\
00010001101101000111011111010010 \\
00010100011100101110010001111101
\end{array}\right],
$$

which can generate $(32,11)$ linear block codes. The minimum code distance of the linear block codes $(32, \mathrm{~A})$ is shown in Table 2, where A means the information length of a linear block code.

Table 2. The minimum distance of the constructed (32, A) codes
\begin{tabular}{|c|c|c|c|}
\hline Information Length & $\mathbf{1}$ & $\mathbf{2} \sim \mathbf{6}$ & $\mathbf{7} \sim \mathbf{1 1}$ \\
\hline Minimum distance & 32 & 16 & 12 \\
\hline
\end{tabular}

\section{Simulation Results}

In this section, we compare the performance of the constructed codes and some widely used codes.

\section{A. $\quad(32, A)$ codes in LTE}

In LTE standard, $(32$, A) linear block codes are used for PUSCH coding [2], and the minimum code distance is

Table 3. The minimum distance of the $(32, \mathrm{~A})$ codes in LTE

\begin{tabular}{|l|c|c|c|c|}
\hline Information Length & $\mathbf{1}$ & $\mathbf{2} \sim \mathbf{6}$ & $\mathbf{7} \sim \mathbf{1 0}$ & $\mathbf{1 1}$ \\
\hline Minimum Distance & 32 & 16 & 12 & $\mathbf{1 0}$ \\
\hline
\end{tabular}


Compare Table 2 and Table 3, we can find that, when the information length is 11 , the minimum distance of our constructed codes is larger than that of the LTE codes by 2 .

Fig. 1 shows the performance comparison of the constructed $(32,11)$ codes and the $(32,11)$ codes in LTE, with QPSK modulation and AWGN channel. Because of the larger minimum distance, the constructed $(32,11)$ code achieves $0.2 \mathrm{~dB}$ performance gain compared to the $(32,11)$ code in LTE.

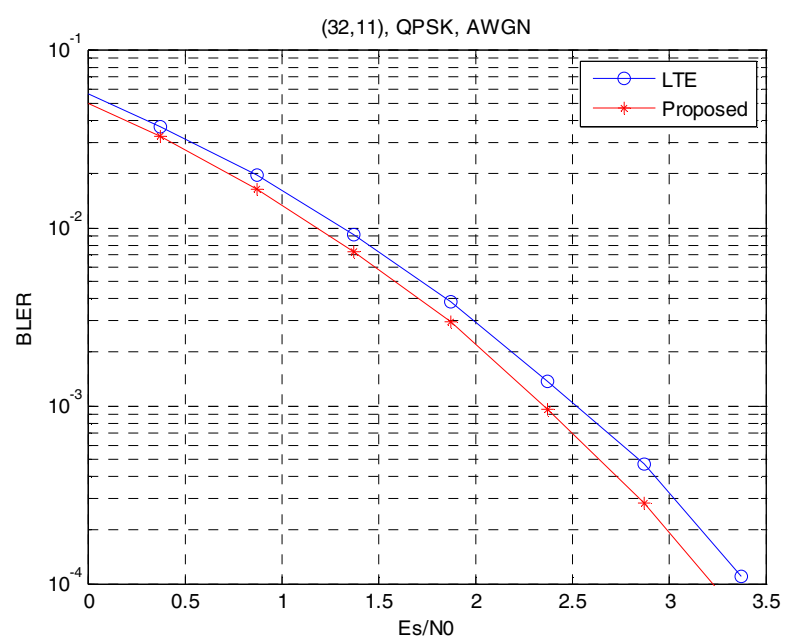

Fig. 1. Performance comparison of the $(32,11)$ codes

B. (40, A) codes for LTE

A $(40$, A) linear block codes was once proposed for LTE standard. These codes can be decoded with low complexity and achieve good performance [21]. Based on our constructed (32, A) codes, we can also construct $(40, A)$ codes by means of simply row repetition. For example, we can add the $\{0,1,5,20$, $22,23,28,31\}$ columns at the right of the $(32, A)$ matrix, and we will get the $(40, \mathrm{~A})$ codes. The columns selection is accomplished by computer search. The minimum distance of the proposed (40, A) codes and the (40, A) codes in Ref.[21] are compared in Table 4.

Table 4. minimum distance comparison of the $(40, A)$ codes

\begin{tabular}{|l|l|l|l|l|l|l|l|l|}
\hline Information Length & $\mathbf{4}$ & $\mathbf{5}$ & $\mathbf{6}$ & $\mathbf{7}$ & $\boldsymbol{8}$ & $\mathbf{9}$ & $\mathbf{1 0}$ & $\mathbf{1 1}$ \\
\hline $\begin{array}{l}\text { Minimum code } \\
\text { Distance of [21] }\end{array}$ & 16 & 16 & 16 & 12 & 12 & 12 & 12 & 8 \\
\hline $\begin{array}{l}\text { Minimum code } \\
\text { Distance of Proposed }\end{array}$ & 20 & 18 & 18 & 14 & 14 & 14 & 13 & 12 \\
\hline
\end{tabular}

We can predict, from the minimum code distance in Table 4 , that the proposed $(40, \mathrm{~A})$ codes should be able to provide better performance than the (40, A) codes in [21], for all different information lengths. We will perform simulations to verify the prediction, under the QPSK modulation and AWGN.
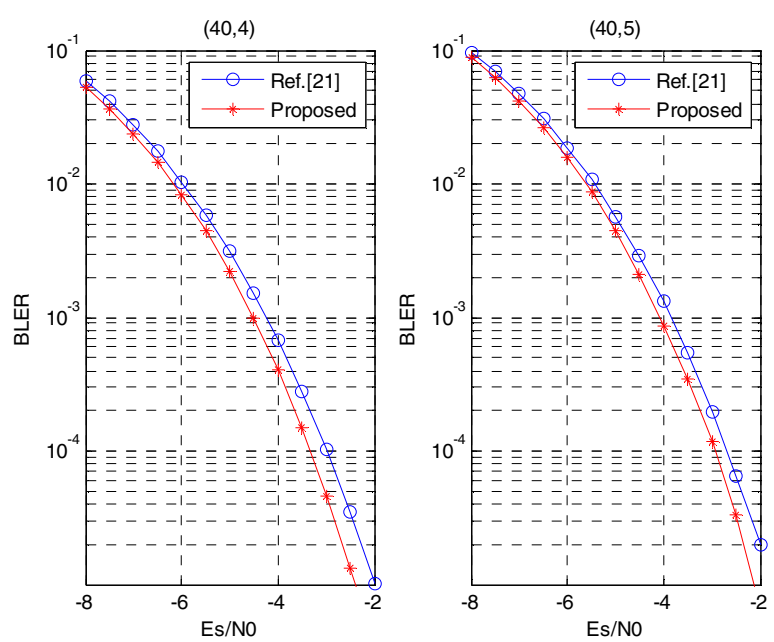

Fig. 2. Performance comparison of $(40,4),(40,5)$ codes
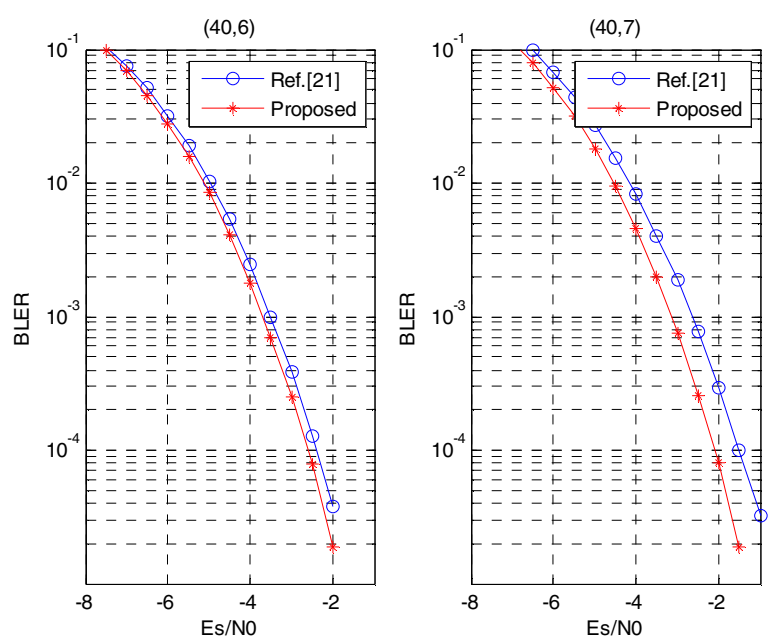

Fig. 3. Performance comparison of $(40,6),(40,7)$ codes
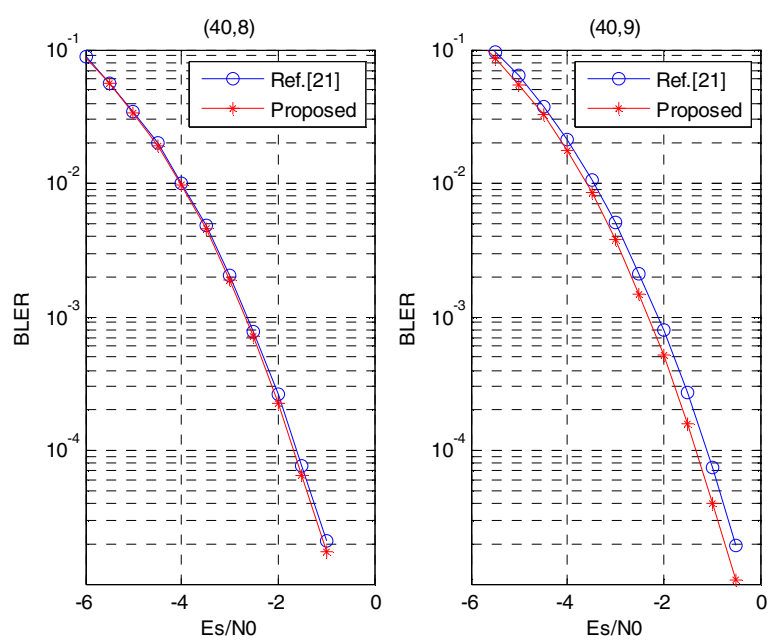

Fig. 4. Performance comparison of $(40,8),(40,9)$ codes 

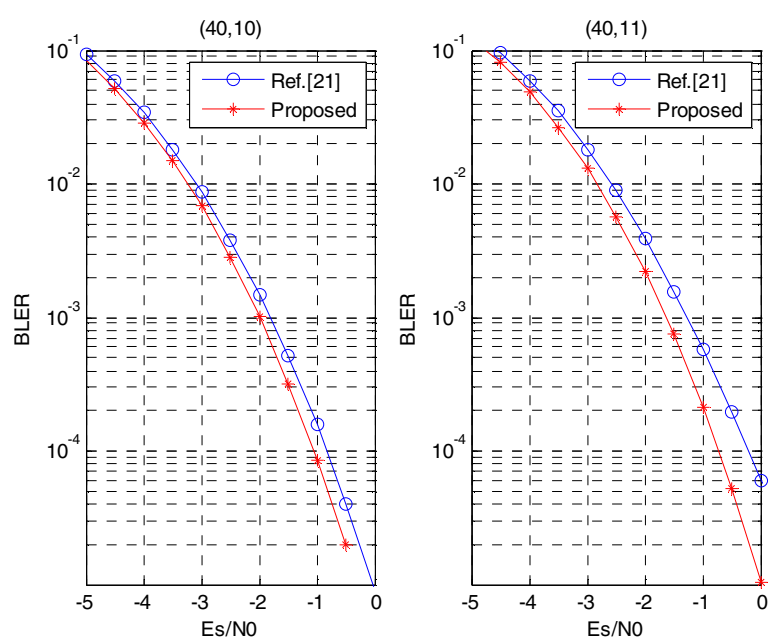

Fig. 5. Performance comparison of $(40,10),(40,11)$ codes

From the simulation results of Fig. 2 to Fig. 5, we can conclude that our proposed $(40$, A) codes can provide better performance than the codes in [21] for all information lengths.

\section{CONCLUSION}

In this paper, we propose a systematic algorithm to construct the super codes of the first order Reed-Muller code based on the m-sequence pairs, which only involves some item permutation within the m-sequence and gets rid of the exhaustive computer search in the conventional code construction. In addition, the constructed super codes outperform the conventional codes in terms of decoding error rate. Simulation result verifies the superiority of our constructed codes.

\section{REFERENCES}

[1] 3GPP, “TS 25.212 v8.5.0”, 2009.6

[2] 3GPP, “TS 36.212 v8.7.0", 2009.6

[3] John G. Proakis, "Digital Communications (Fourth Edition)", 2003

[4] 3GPP R1-080575, "Way Forward on PUCCH CQI coding". LGE, 2008

[5] Paterson " Generalized Reed-Muller code and Power Control in OFDM Modulation " IEEE Transactions on Information Theory, Vol. 46, No. 1, January 2000, pp. 104-120
[6] K. -U. Schmidt, "On cosets of the generalized first-order Reed-Muller code with low PMEPR,” IEEE Trans. Inform. Theory, vol. 52, no. 7, pp. 3220-3232

[7] Kaustuvmani Manji; Sundar Rajan, B, "On the PAPR of binary ReedMuller OFDM codes”, ISIT2004, pp. 423

[8] C. Fontaine, On some cosets of the first-order Reed-Muller code with high minimum Weight, IEEE Trans. Inform. Theory, Vol. 45(4) (1999) pp. 1237-1243

[9] E. R. Berlekamp and L. R. Welch, "Weight distributions of the cosets of the $(32 ; 6)$ Reed-Muller code," IEEE Trans. Inform. Theory, vol. IT-18, pp. 203-207, Jan. 1972.

[10] KASAMI Tadao, FUJIWARA Toru, and DESAKI Yoshihisa; "The Weight Distributions of Cosets of the Second-Order Reed-Muller Code of Length 128 in the Third-Order Reed-Muller Code of Length 128", IEICE transactions on fundamentals, VOL. E79-A, No. 4, April 1996

[11] S. W. Golomb, Shift Register Sequences. San Francisco: Holden-Day, 1967.

[12] E. R. Berlekamp, Algebraic Coding Theory. New York: McGraw-Hill, 1968

[13] P. Dklsarte, J. M. Goethals, and F. J. MacWilliams, "On generalized Reed-Muller code and their relatives, "Information and Control, vol. 16, pp. 403-142, July 1970.

[14] Cohn, M. Lempel, A. "On fast M-sequence transforms", IEEE Transactions on Information Theory, Volume 23, Issue 1, pp. 135- 137, Jan 1977

[15] Wang Shichang, Wang Yankun, Tong Yufeng, "m Sequences and Hadamard Matrix", JOURNAL OF YANTAI UNIVERSITY, No. 1, pp. 7-11, 1998

[16] QIU Qing, XIN Gang, ZHANG Shui lian, "Frame Synchronization in an M-ary Orthogonal Coded Spread Spectrum System", Journal of Information Engineering University, No. 2, pp. 75-77, 2003

[17] R. Gold, "Maximal recursive sequences with 3-valued recursive crosscorrelation functions," IEEE Trans. Inf. Theory, vol. IT-14(1), pp. 154-156, Jan. 1968

[18] S. Boztas and P. V. Kumar, "Binary sequences with Gold-like correlation but larger linear span," IEEE Trans. Inf. Theory, vol. 40(2), pp. 532-537, Mar. 1994.

[19] J.H. Conway and N.J.A. Sloane, "Soft Decoding Techniques for Codes and Lattices, Including the Golay Code and the Leech Lattice", IEEE Trans. Inform. Theory, vol.32, pp.41-50, Jan 1986

[20] Yair Be'ery, Jakov Snyders: Optimal soft decision block decoders based on fast Hadamard transform. IEEE Transactions on Information Theory 32(3): 355-364 (1986)

[21] 3GPP R1-081263, "Detail of CQI coding on PUSCH piggybacked from PUCCH”. LGE, 2008 